\title{
Effet du biochar sur la biodisponibilité du phosphore dans un sol limoneux acide
}

\author{
David Houben ${ }^{(1)}$, Brieuc Hardy ${ }^{(2)}$, Michel-Pierre Faucon ${ }^{(1)}$, Jean-Thomas Cornelis ${ }^{(3)}$ \\ (1) UniLaSalle. HydrISE. 19, rue Pierre Waguet. F-60026 Beauvais cedex (France).E-mail : david.houben@unilasalle.fr \\ (2) Université catholique de Louvain. Earth and Life Institute - Environmental Sciences. Croix du Sud, 2 bte L7.05.10. \\ BE-1348 Louvain-la-Neuve (Belgique). \\ (3) Université de Liège - Gembloux Agro-Bio Tech. Département Biosystem Engineering (BIOSE). 27, Avenue Maréchal \\ Juin. BE-5030 Gembloux (Belgique).
}

Reçu le 4 avril 2016, accepté le 14 mars 2017

Cet article est distribué suivant les termes et les conditions de la licence CC-BY (http://creativecommons.org/licenses/by/4.0/ deed.fr)

Description du sujet. Cet article traite de l'impact du biochar sur la biodisponibilité en phosphore (P) dans les sols en vue d'améliorer la gestion et l'autonomie de la fertilisation en P des cultures.

Objectifs. L'objectif général était d'explorer le potentiel du biochar à augmenter la biodisponibilité du P dans le sol. Les objectifs spécifiques étaient de préciser l'influence de la biomasse pyrolysée ainsi que la dose de biochar appliquée sur la solubilité du P.

Méthode. Trois biochars produits à partir de biomasses différentes (résidus de Miscanthus, de bois et de café) ont été incorporés dans un Luvisol ( $\mathrm{pH}$ acide) selon deux doses ( 1 et $3 \%$ en masse). Après 76 jours d'incubation, la biodisponibilité du $\mathrm{P}$ a été estimée (extraction au $\mathrm{CaCl}_{2} 0,01 \mathrm{M}$ ). Les propriétés physico-chimiques du sol et la quantité de $\mathrm{CO}_{2}$ émise durant la période d'incubation ont également été déterminées.

Résultats. Seul le biochar produit à partir de résidus de bois et incorporé à une dose de $3 \%$ a augmenté la concentration en $\mathrm{P}$ biodisponible dans le sol $(+75 \%)$. Cette augmentation résulterait non seulement d'une libération de $\mathrm{P}$ par le biochar lui-même (effet direct), mais également d'une remobilisation du P du sol (effet indirect) faisant suite à l'élévation drastique du pH (+ 3,6 unités) ainsi qu'à l'augmentation de l'activité biologique. Pour les autres traitements, l'absence d'effet significatif sur la biodisponibilité du P résulte vraisemblablement de leur faible impact sur le $\mathrm{pH}$ du sol, celui-ci restant dans une gamme $(4,3-5,1)$ favorisant l'insolubilisation du P.

Conclusions. Étant donné la variabilité des résultats et les incertitudes concernant les mécanismes responsables de la mobilisation du P en présence de biochar, il est essentiel de conduire des études complémentaires afin de mieux comprendre l'impact du biochar sur la mobilité du P dans les systèmes sol-plante.

Mots-clés. Phosphore, fertilité du sol, biodisponibilité, séquestration du carbone.

Effect of biochar on phosphorus bioavailability in an acidic silt loam soil

Description of the subject. This paper deals with the impact of biochar on soil phosphorus (P) bioavailability with a view to improving the management of $\mathrm{P}$ fertilization.

Objectives. The aim of this study was to explore the potential of biochar to increase P bioavailability in soil. The specific objectives were to elucidate the role of feedstock and the rate of application of biochar on P solubility.

Method. Three biochars produced from different feedstocks (Miscanthus straws, coffee husks and woody material) were added to an acidic Luvisol at two rates of application (1\% and 3\%; w/w). At the end of a 76-day incubation period, P bioavailability was assessed $\left(0.01 \mathrm{M} \mathrm{CaCl}_{2}\right.$ extraction). Soil physico-chemical properties and the amount of $\mathrm{CO}_{2}$ emitted over the incubation period were also determined.

Results. The wood-derived biochar applied at 3\% was the only treatment that increased significantly P bioavailability $(+75 \%)$. This increase might result from the release of $\mathrm{P}$ by biochar itself (direct effect) but also from an enhanced P solubility in soil (indirect effect) related to a large increase in $\mathrm{pH}$ (+3.6 units compared to the control) and a higher soil biological activity. The other treatments had no significant impact on soil $\mathrm{P}$ bioavailability, probably as a result of their minor effect on soil $\mathrm{pH}$. 
Conclusions. Our study shows that biochar-induced changes in P bioavailability in soil varied greatly with type of feedstock and rate of application. However, the balance between the direct and indirect effects of biochar on P bioavailability was not elucidated. Further investigations are thus essential to clarify the potential of biochar to improve P bioavailability in the soil-plant system.

Keywords. Phosphorus, soil fertility, bioavailability, carbon sequestration.

\section{INTRODUCTION}

Le phosphore $(\mathrm{P})$ est un élément essentiel à toute forme de vie. Toutefois, bien qu'il soit naturellement présent dans les sols, les exportations répétées de $\mathrm{P}$ par les récoltes engendrent inévitablement une diminution du stock de $\mathrm{P}$ biodisponible. Compte tenu de son caractère limitant pour la production agricole, le maintien de rendements élevés permettant de garantir la sécurité alimentaire ne peut être réalisé que si ces exportations sont compensées par des apports de fertilisants. Le $\mathrm{P}$ des fertilisants minéraux provient principalement de l'extraction minière des gisements fossiles de phosphates naturels. Cette ressource est disponible en quantité limitée et non renouvelable. Il est en effet prédit que les réserves économiquement exploitables seront épuisées d'ici un à deux siècles (Cordell et al., 2009 ; Van Vuuren et al., 2010), alors même que la pratique de fertilisation phosphatée est relativement récente, celle-ci s'étant répandue après la Première Guerre mondiale (Boulaine, 2006). La nécessité de gérer durablement cette ressource minière, conjuguée aux menaces environnementales causées par un excès de $\mathrm{P}$ dans les agroécosystèmes (ex : eutrophisation des eaux continentales), impose le développement de meilleures pratiques de gestion de la fertilisation phosphatée (Fixen \& Johnston, 2012). Cela passe notamment par la conception de systèmes de production agricole dans lesquels la biodisponibilité du $\mathrm{P}$ dans les sols est accrue et les apports de fertilisants minéraux sont limités ou substitués par des intrants phosphatés issus de ressources renouvelables (Faucon et al., 2015).

La récupération du $\mathrm{P}$ des sous-produits organiques sous forme de biochar a récemment été suggérée comme une stratégie alternative prometteuse pour réduire l'utilisation des engrais minéraux (Azuara et al., 2013 ; Atienza-Martínez et al., 2014 ; Zhai et al., 2015). Le biochar est une substance poreuse riche en carbone produite par un procédé appelé pyrolyse qui consiste à décomposer un matériau organique (ex : bois, déchets verts, boues, etc.) par voie thermochimique et sous apport limité d'oxygène $\left(\mathrm{O}_{2}\right)$ (Lehmann \& Joseph, 2009). Afin de le différencier du charbon de bois, la définition adoptée par la International Biochar Initiative (IBI) spécifie que la finalité de cette substance est son utilisation en tant qu'amendement pour les sols (Sohi et al., 2010). Depuis quelques années, le biochar suscite l'engouement de la communauté scientifique qui le considère comme un moyen permettant de lutter contre le changement climatique global tout en améliorant la fertilité des sols (Lehmann et al., 2006). En effet, le biochar est un produit chimiquement et biologiquement récalcitrant dont le temps de résidence dans les sols peut atteindre plus de 1000 ans (Lehmann, 2007). Dès lors, l'incorporation de biochar dans le sol permet de séquestrer du carbone atmosphérique fixé photosynthétiquement par la biomasse. Par ailleurs, l'apport de biochar dans le sol augmente généralement la rétention en éléments nutritifs, notamment grâce à sa surface spécifique élevée couplée à la présence de nombreux groupes fonctionnels superficiels qui accroissent la capacité d'échange cationique (CEC) du système sol-biochar (Sohi et al., 2010 ; Verheijen et al., 2010 ; Kookana et al., 2011). Il a également été démontré que son application pouvait améliorer la rétention en eau utile, diminuer la toxicité de certains polluants et favoriser la biodiversité de la macro- et micro-faune dans les sols (Lehmann et al., 2011; Houben et al., 2013a ; Houben et al., 2013b ; Rees et al., 2014). En conséquence, un accroissement de la productivité des cultures est habituellement observé en présence de biochar (Manyà, 2012; Biederman \& Harpole, 2013), même si d'autres études mettent également en évidence des effets plus nuancés (Jeffery et al., 2011) ou suggèrent un potentiel impact négatif sur certaines propriétés agronomiques du sol (augmentation de la salinité, enrichissement en contaminants, etc.). Enfin, en plus d'améliorer la fertilité des sols et la séquestration de carbone, deux bénéfices additionnels peuvent motiver la production de biochar : la valorisation de déchets organiques et la production d'énergie récupérable lors de la pyrolyse (bio-huile et syngaz) (Yaman, 2004), ce qui contribue à réduire davantage les émissions de gaz à effet de serre à partir des énergies fossiles (Gaunt \& Lehmann, 2008).

Puisque le $\mathrm{P}$ ne se volatilise qu'à partir de 700$800{ }^{\circ} \mathrm{C}$, c'est-à-dire à des températures supérieures à celles rencontrées lors de la pyrolyse, la totalité du $\mathrm{P}$ présent dans la matière première se retrouve dans le biochar, contrairement au $\mathrm{C}$ organique dont une fraction se volatilise à partir de $100^{\circ} \mathrm{C}$ sous forme de $\mathrm{CO}$, de $\mathrm{CO}_{2}$ et de composés organiques volatils (Lide, 2004). La pyrolyse concentre ainsi le P dans le résidu solide. Par ailleurs, elle provoque la rupture des liaisons organiques phosphatées, aboutissant à la formation d'un sel phosphaté soluble associé au 
matériau carbonisé. Par conséquent, la biodisponibilité du P dans le biochar est plus élevée que celle dans la biomasse non pyrolysée (DeLuca et al., 2009). Bien que toute matière organique puisse en principe être pyrolysée, les propriétés physico-chimiques du biochar peuvent considérablement varier selon les conditions pyrolytiques (principalement la température, la vitesse de chauffe et le temps de résidence à l'intérieur du réacteur) et le type de matière première pyrolysée (Chan \& Xu, 2009; Verheijen et al., 2010). Par conséquent, l'impact du biochar sur la biodisponibilité du P dans les sols est susceptible de varier en fonction des conditions de pyrolyse et du type de matière organique pyrolysée. De plus, l'utilisation d'un même biochar à différentes doses peut engendrer des effets diamétralement opposés sur la productivité des cultures et les cycles des éléments nutritifs (Jeffery et al., 2011; Biederman $\&$ Harpole, 2013). Comme la plupart des amendements organiques, l'impact du biochar sur la biodisponibilité des éléments nutritifs dans le sol résulte à la fois d'effets directs (i.e. libération d'éléments nutritifs par le biochar lui-même) et indirects (e.g. modifications physico-chimiques et biologiques du milieu) (Faucon et al., 2015). Ces effets peuvent par ailleurs être euxmêmes modulés par les propriétés initiales du sol. Par exemple, en raison du caractère habituellement alcalin du biochar, son impact sur la biodisponibilité des éléments en sol acide peut être contraire à celui observé en sol alcalin (Biederman \& Harpole, 2013). En prérequis à l'incorporation de biochar dans les sols agricoles, il est dès lors crucial d'élucider l'impact du type de biomasse pyrolysée sur la biodisponibilité du P, d'identifier les taux d'applications du biochar optimaux et de déterminer les mécanismes pouvant impacter le comportement du $\mathrm{P}$ après apport de biochar dans le sol. La présente étude ambitionne d'évaluer le potentiel de biochars produits dans des conditions pyrolytiques identiques à partir de trois biomasses différentes (résidus de Miscanthus, de bois et de café) et apportés à deux doses (1 et $3 \%$ en masse) à améliorer la biodisponibilité du $\mathrm{P}$ dans le sol.

\section{MATÉRIEL ET MÉTHODES}

\subsection{Sol}

Afin de pouvoir détecter l'effet du biochar sur la biodisponibilité du P, la sélection du sol de cette étude s'est portée sur un sol non fertilisé et non chaulé. L'échantillonnage du sol a été réalisé au sein de l'horizon argique (Bt) d'un Luvisol localisé sous forêt dans la ceinture loessique du centre de la Belgique. Le sol échantillonné est caractérisé par un $\mathrm{pH}$ (ratio sol:eau $=1: 5)$ de 4,3, une CEC de 7,9 $\mathrm{cmol}_{\mathrm{c}} \cdot \mathrm{kg}^{-1}$ et une teneur en $\mathrm{C}$ organique de $0,43 \%$ (Houben et al.,
2014). Après échantillonnage, le sol a été séché à température ambiante, tamisé à $2 \mathrm{~mm}$ et stocké à $20^{\circ} \mathrm{C}$ dans l'obscurité.

\subsection{Biochars}

Les biochars ont été obtenus auprès de l'entreprise Pyreg GmbH (Dörth, Allemagne). Trois types de biochar ont été produits à partir d'enveloppes de café (appelé ci-après BiocharCafé), de résidus de bois (appelé ci-après BiocharBois) et de paille de Miscanthus (appelé ci-après BiocharMiscanthus). Les trois biochars ont été produits dans des conditions de production identiques: le temps de résidence dans le réacteur était de $30 \mathrm{~min}$ et la température finale de $600{ }^{\circ} \mathrm{C}$. Le $\mathrm{pH}$, la teneur en $\mathrm{C}$ (déterminée par combustion sèche au moyen d'un analyseur CNS analyser; Flash EA 1112 Series) ainsi que la teneur en P (déterminée après fusion alkaline) (Chao \& Sanzolone, 1992) de chacun des biochars sont présentés dans le tableau 1. Les trois biochars présentent des concentrations en $\mathrm{P}$ relativement similaires entre elles et situées dans la gamme des valeurs habituellement rapportées (Chan \& Xu, 2009; Schneider \& Haderlein, 2016). Les particules de biochar ont également été observées et leur composition élémentaire a été déterminée semi-quantitativement au microscope électronique à balayage (MEB ; Zeiss Utra55) équipé d'un canon à effet de champ (field emission gun; FEG) et d'un système d'analyse par dispersion d'énergie (energydispersive $X$-ray spectrometer; EDS). La tension a été fixée à $20 \mathrm{kV}$.

\subsection{Préparation des traitements et expérience d'incubation}

Le sol et les mélanges sol-biochar utilisés dans cette étude proviennent d'une expérience d'incubation réalisée par Houben et al. (2014) et qui visait à l'étude de l'amélioration de la biodisponibilité du silicium ( $\mathrm{Si}$ ) combinée à la séquestration de $\mathrm{C}$ dans les sols grâce

Tableau 1. Principales caractéristiques des biochars (d'après Houben et al., 2014) produits à partir d'enveloppes de café (BiocharCafé), de résidus de bois (BiocharBois) et de paille de Miscanthus (BiocharMiscanthus) - Main characteristics of biochars (data from Houben et al., 2014) produced from coffee grounds (BiocharCafé), woody materials (BiocharBois) and Miscanthus straws (BiocharMiscanthus).

\begin{tabular}{llll}
\hline & $\mathbf{p H}$ & $\mathbf{C}(\%)$ & $\mathbf{P}\left(\mathrm{mg} \cdot \mathrm{kg}^{-1}\right)$ \\
\hline BiocharCafé & 10,1 & 76,6 & 3900 \\
BiocharBois & 10,7 & 66,4 & 4000 \\
BiocharMiscanthus & 10,1 & 52,8 & 2500 \\
\hline
\end{tabular}


à un apport de biochar. Brièvement, les mélanges sol-biochar ont été préparés et homogénéisés en incorporant une dose de $1 \%$ ou $3 \%$ (rapport massique) de chaque biochar à des sous-échantillons de sol. Ces doses correspondent à celles fréquemment rencontrées dans la littérature (Biederman \& Harpole, 2013). Vingt grammes de sol et des mélanges sol-biochar ont été incubés à l'obscurité à $20^{\circ} \mathrm{C}$ dans des flacons hermétiques pendant 76 jours, à raison de trois réplicats par traitement. La teneur en eau de chaque traitement a été ajustée à la capacité au champ. Le $\mathrm{CO}_{2}$ dégagé au cours de l'incubation a été piégé dans $25 \mathrm{ml}$ de soude $(\mathrm{NaOH}, 0.497 \mathrm{M})$ et dosé par la méthode développée par Rodella \& Saboya (1999). Pour comparer les différents traitements, la quantité de $\mathrm{C}$ émis sous forme de $\mathrm{CO}_{2}\left(\mathrm{C}-\mathrm{CO}_{2}\right)$ a ensuite été rapportée par la masse de sol incorporé dans chacun des flacons.

\subsection{Analyses physico-chimiques}

Le sol et les mélanges sol-biochar collectés à la fin de l'expérience d'incubation ont été séchés à température ambiante. Leur $\mathrm{pH}$ a été déterminé dans une suspension sol-eau (ratio massique 5:25). La concentration en $\mathrm{P}$ biodisponible a été estimée au moyen d'une extraction au $\mathrm{CaCl}_{2}$ (Houba et al., 2000). Cette extraction a précédemment été suggérée pour estimer la quantité de $\mathrm{P}$ facilement utilisable par les cultures (McDowell et al., 2002 ; Devau et al., 2009). Brièvement, après avoir agité $10 \mathrm{~g}$ de sol dans $100 \mathrm{ml}$ d'une solution de $\mathrm{CaCl}_{2} 0,01 \mathrm{M}$ pendant $5 \mathrm{~h}$, la concentration en $\mathrm{P}$ dans le surnageant récupéré après centrifugation $(3000 \mathrm{~g}$; $15 \mathrm{~min}$ ) et filtration (Whatman $\mathrm{n}^{\circ} 40$ ) a été déterminée par spectrométrie d'émission à source plasma à couplage induit (Inductively coupled plasma atomic emission spectroscopy ; ICP-AES). Afin d'estimer la capacité du sol à réalimenter la solution du sol en $\mathrm{P}$, l'extraction a été répétée six fois (McDowell \& Sharpley, 2003). La concentration en C organique dissous (COD) dans le surnageant de la première extraction a été déterminée au moyen d'un analyseur de C (Dohrmann DC-180C).

\subsection{Analyses statistiques}

Les statistiques descriptives ont été utilisées pour quantifier la variabilité des données obtenues. Ces statistiques intègrent le calcul de la moyenne et de l'erreur standard. La normalité et l'homoscédasticité des données ont été vérifiées. Pour les distributions asymétriques, les données ont été log-transformées pour répondre aux hypothèses de l'analyse de variance (ANOVA). Une ANOVA à deux facteurs (two-way ANOVA) a été réalisée afin de déterminer l'effet du type de biochar, de son taux d'application et de leur interaction sur les paramètres étudiés. Le test de Tukey a été utilisé pour comparer les moyennes lorsque l'ANOVA a révélé des différences significatives entre les traitements au seuil de probabilité de $5 \%$. Toutes les analyses statistiques ont été réalisées au moyen du logiciel R.

\section{RÉSULTATS ET DISCUSSION}

\subsection{Impact du biochar sur la biodisponibilité du $P$ dans le sol}

Les résultats de l'ANOVA à deux facteurs (Tableau 2) indiquent que le type et la dose de biochar et l'interaction de ces facteurs ont un effet significatif sur la biodisponibilité en $\mathrm{P}$ du sol. Toutefois, par rapport au traitement sans biochar, seul le traitement BiocharBois-3\% a engendré une augmentation significative de la biodisponibilité du $\mathrm{P}$ dans le sol à l'issue de la période d'incubation (Figure 1). De plus, seule l'application de biochar de bois à $3 \%$ semble avoir impacté la dynamique de libération du $\mathrm{P}$ (Figure 2), suggérant que la libération du $\mathrm{P}$ dans les autres traitements est essentiellement gouvernée par les

Tableau 2. Résultats de l'ANOVA à deux facteurs testant l'effet du type de biochar, de la dose apportée et de leur interaction sur la concentration en phosphore biodisponible (première étape de l'extraction au $\mathrm{CaCl}_{2} 0,01 \mathrm{M}$ ), le $\mathrm{pH}$, la concentration en $\mathrm{C}$ organique dissous ( $\mathrm{COD}$; extraction au $\mathrm{CaCl}_{2}, 0,01 \mathrm{M}$ ) et la quantité totale de $\mathrm{C}-\mathrm{CO}_{2}$ émis à l'issue de la période d'incubation (76 jours) - Two-way ANOVA results testing the effects of biochar type, application rate and their interaction on the concentration of available phosphorus (first step of the $0.01 \mathrm{M} \mathrm{CaCl}_{2}$ extraction), pH, concentration of dissolved organic carbon ( $\mathrm{COD} ; 0.01 \mathrm{M} \mathrm{CaCl}_{2}$ extraction) and total amount of $\mathrm{CO}_{2}$ - $\mathrm{C}$ released after 76 days of incubation.

\begin{tabular}{|c|c|c|c|c|c|c|c|c|c|c|c|c|}
\hline & \multicolumn{4}{|c|}{ Type de biochar } & \multicolumn{4}{|c|}{ Dose apportée } & \multicolumn{4}{|c|}{ Type $x$ dose } \\
\hline & Sum Sq & Df & $\mathbf{F}$ & $\operatorname{Pr}(>F)$ & Sum Sq & Df & $\mathbf{F}$ & $\operatorname{Pr}(>F)$ & Sum Sq & Df & $\mathbf{F}$ & $\operatorname{Pr}(>F)$ \\
\hline $\mathrm{P}_{\mathrm{CaCl}_{2}}$ & 0,140 & 2 & 4,22 & $<0,050$ & 0,550 & 2 & 16,36 & $<0,001$ & 0,410 & 2 & 12,06 & $<0,001$ \\
\hline $\mathrm{pH}$ & 10,020 & 2 & 274,63 & $<0,001$ & 20,880 & 2 & 572,25 & $<0,001$ & 6,310 & 2 & 172,91 & $<0,001$ \\
\hline COD & 4201,500 & 2 & 66,57 & $<0,001$ & 7215,50 & 2 & 114,33 & $<0,001$ & 275,100 & 2 & 4,36 & $<0,050$ \\
\hline $\mathrm{C}-\mathrm{CO}_{2}$ & 0,013 & 2 & 1,77 & 0,210 & 0,067 & 2 & 8,84 & $<0,010$ & 0,032 & 2 & 4,19 & $<0,050$ \\
\hline
\end{tabular}




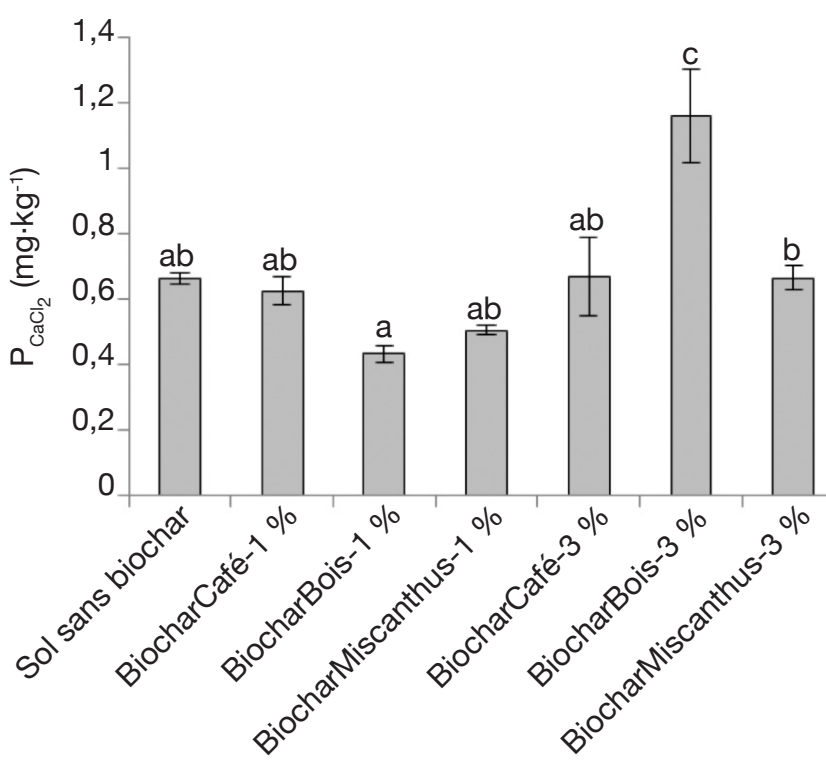

Figure 1. Concentration en phosphore biodisponible (première étape de l'extraction au $\mathrm{CaCl}_{2} 0,01 \mathrm{M}$ ) dans le sol non traité (sol sans biochar) et dans le sol amendé avec $1 \%$ ou $3 \%$ de biochar produit à partir d'enveloppes de café (BiocharCafé), de résidus de bois (BiocharBois) et de paille de Miscanthus (BiocharMiscanthus) - Concentration of available phosphorus (first step of the $0.01 \mathrm{M} \mathrm{CaCl}_{2}$ extraction) in the untreated soil and in the soil amended with $1 \%$ or $3 \%$ of biochar produced from coffee grounds (BiocharCafé), woody materials (BiocharBois) and Miscanthus straws (BiocharMiscanthus).

Les résultats représentent la moyenne de trois réplicats \pm l'erreur standard - results are mean of three replicates \pm standard deviation; les moyennes surmontées de la même lettre alphabétique ne diffèrent pas significativement au seuil de $5 \%$ selon le test de Tukey - columns with same letter do not differ significantly at the 5\% level according to the Tukey's multiple comparison test.

propriétés intrinsèques du sol. Ces résultats indiquent que l'effet du biochar sur la biodisponibilité en $\mathrm{P}$ dans le sol dépend non seulement de la matière première à partir duquel il a été produit mais également du taux auquel il est appliqué.

\subsection{Mécanismes potentiellement impliqués dans l'immobilisation/la mobilisation du P en présence de biochar}

L'absence d'effet significatif des traitements BiocharCafé-1 $\%$ et -3\%, BiocharMiscanthus-1\% et $-3 \%$ et BiocharBois-1\% sur la biodisponibilité du P par rapport au contrôle résulte probablement du faible impact de ces biochars sur le pH du sol (Figure 3). En effet, bien que ces traitements augmentent significativement le $\mathrm{pH}$ du sol (effet significatif du type de biochar, de la dose apportée et de l'interaction de
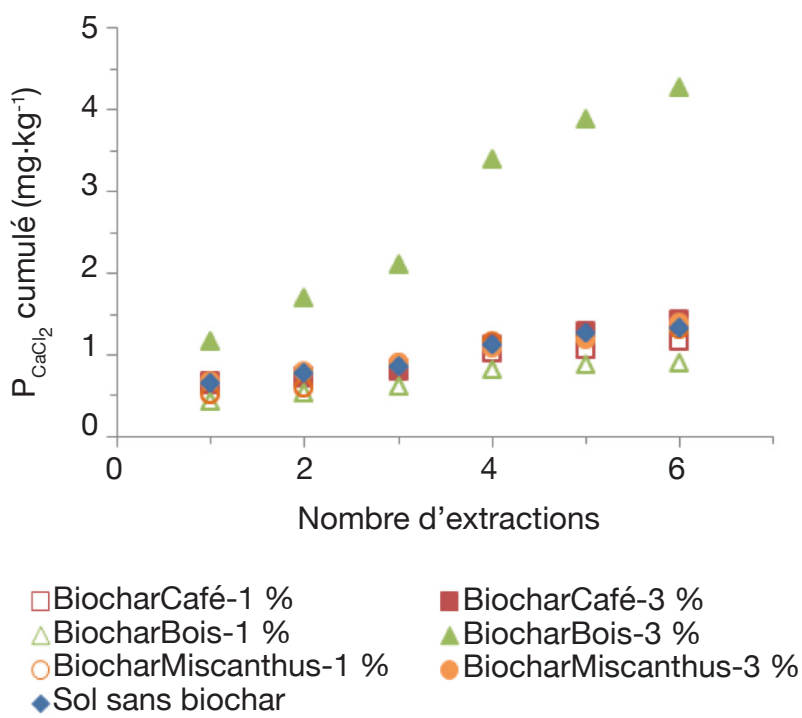

Figure 2. Libération du phosphore (extrait au $0,01 \mathrm{M} \mathrm{CaCl}_{2}$ ) du sol et des mélanges sol-biochar - Phosphorus release $\left(\mathrm{CaCl}_{2} 0.01 \mathrm{M}\right.$ extraction) in the untreated soil and in the soil-biochar mixtures.

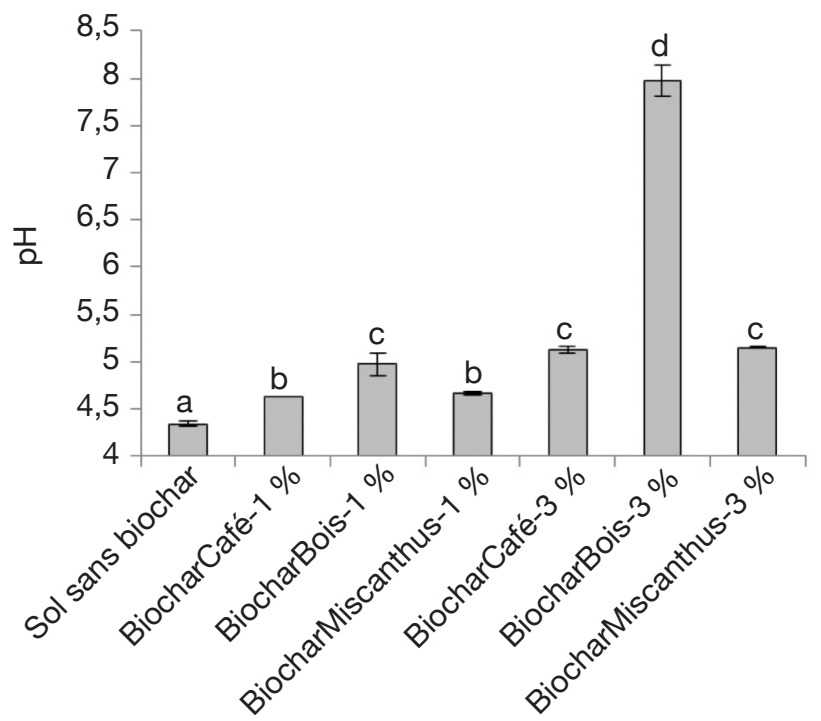

Figure 3. pH du sol non traité et des mélanges sol-biochar à l'issue de la période d'incubation $-p H$ in the untreated soil and in the soil-biochar mixtures after incubation.

Les résultats représentent la moyenne de trois réplicats \pm l'erreur standard - results are mean of three replicates \pm standard deviation; les moyennes surmontées de la même lettre alphabétique ne diffèrent pas significativement au seuil de $5 \%$ selon le test de Tukey - columns with same letter do not differ significantly at the 5\% level according to the Tukey's multiple comparison test. 
ces deux facteurs; Tableau 2), ce dernier reste dans une gamme de valeurs au sein de laquelle le $\mathrm{P}$ est très peu biodisponible en raison de sa forte sorption par les oxyhydroxydes de $\mathrm{Fe}$ et d'Al et les minéraux argileux (Houben et al., 2011 ; Gérard, 2016). Plus spécifiquement, McDowell et al. (2003) ont montré que la solubilité du $\mathrm{P}_{\mathrm{CaCl}_{2}}$ était fortement réduite dans des sols présentant des valeurs de $\mathrm{pH}$ inférieures à 5,8 suite à la formation de variscite $\left(\mathrm{AlPO}_{4} \cdot 2 \mathrm{H}_{2} \mathrm{O}\right)$, un minéral très stable et peu soluble. Exception faite du BiocharBois-3 \%, le P libéré par le biochar dans les différents traitements a donc été rapidement fixé par les constituants du sol, limitant dès lors le pouvoir fertilisant phosphaté de ces biochars.

Contrairement aux autres traitements, le BiocharBois-3\% a engendré une augmentation significative de la biodisponibilité en $\mathrm{P}$, les concentrations en $\mathrm{P}_{\mathrm{CaCl}}$ augmentant de $75 \%$ par

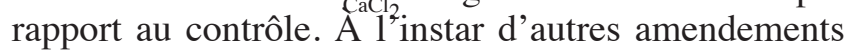
organiques, l'incorporation de biochar dans des sols acides peut accroitre la biodisponibilité du $\mathrm{P}$ non seulement en approvisionnant le sol en $\mathrm{P}$ (effet direct), mais également en favorisant sa mise en solution (effet indirect). La solubilité du P du sol peut en effet augmenter après de tels apports suite à :

- l'augmentation du pH du sol qui diminue la capacité de fixation du sol vis-à-vis du $\mathrm{P}$ (diminution de la quantité de charges positives responsables de l'adsorption du $\mathrm{P}$ par déprotonation des oxyhydroxides de $\mathrm{Fe}$ et $\mathrm{Al}$, ce qui augmente la quantité de charges négatives et le phénomène de répulsion entre les anions phosphates et les constituants du sol) (Murrmann \& Peech, 1969 ; Devau et al., 2010) et favorise la dissolution des phosphates de fer et d'aluminium tels que la variscite ou son homologue amorphe (McDowell et al., 2003) ;

- la libération d'anions organiques qui entrent en compétition pour les mêmes sites de fixations que le $\mathrm{P}$ (échange de ligands) et complexent les ions métalliques $(\mathrm{Ca}, \mathrm{Al}, \mathrm{Fe})$ impliqués dans l'immobilisation du P (Ohno \& Crannell, 1996; Cong \& Merckx, 2005 ; Faucon et al., 2015).

Les données de la présente étude ne permettent pas de distinguer les effets directs des effets indirects. Toutefois, les concentrations en $\mathrm{C}$ organique dissous (extraction au $\mathrm{CaCl}_{2} 0,01 \mathrm{M}$ ) étant les plus faibles pour le traitement BiocharBois-3 \% (Figure 4), l'hypothèse d'une mobilisation accrue du $\mathrm{P}$ suite à une plus forte concentration en anions organiques peut être exclue. En revanche, l'augmentation drastique $\mathrm{du} \mathrm{pH}$ après l'incorporation du BiocharBois-3\% (+ 3,6 unités par rapport au sol sans biochar ; Figure 3) a certainement contribué à accroitre la biodisponibilité du $\mathrm{P}$ dans le sol. Cette augmentation de $\mathrm{pH}$ est cohérente à l'effet chaulant habituellement reporté pour le biochar

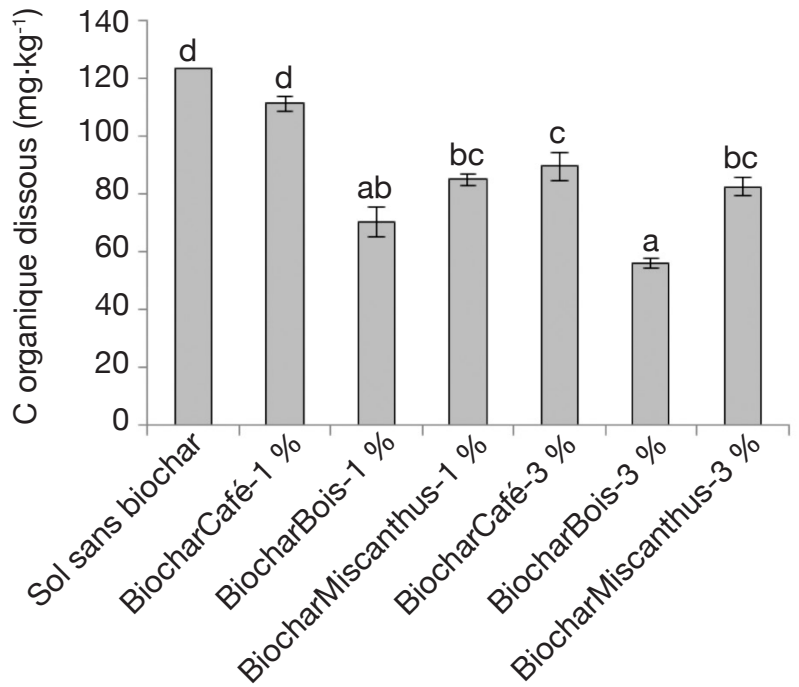

Figure 4. Concentration en carbone organique dissous dans le sol et les mélanges sol-biochar à l'issue de la période d'incubation (extraction au $\mathrm{CaCl}_{2} 0,01 \mathrm{M}$ ) - Concentration of dissolved organic carbon in the untreated soil and in the soil-biochar mixtures after incubation.

Les résultats représentent la moyenne de trois réplicats \pm l'erreur standard - results are mean of three replicates \pm standard deviation; les moyennes surmontées de la même lettre alphabétique ne diffèrent pas significativement au seuil de $5 \%$ selon le test de Tukey - columns with same letter do not differ significantly at the 5\% level according to the Tukey's multiple comparison test.

(Joseph et al., 2010 ; Houben \& Sonnet, 2015) et résulte de la dissolution d'oxydes, hydroxydes et carbonates de métaux alcalins et alcalino-terreux (ex : $\mathrm{CaO}, \mathrm{CaCO}_{3}$ ) associés au biochar (Yuan et al., 2011 ; Hass et al., 2012). L'apport de composés alcalinisants est par ailleurs confirmé par la présence de cristaux de calcite au sein du biochar produit à partir de résidus de bois (Figure 5). De tels composés n'ont pas été observés par microscopie électronique à balayage dans les deux autres biochars. Nos résultats rejoignent ceux obtenus par d'autres études récentes qui mettent en évidence le rôle prépondérant joué par l'alcalinisation du sol subséquente à une application de biochar sur la biodisponibilité du P dans le sol (Chintala et al., 2014). Il convient toutefois de noter que dans des sols non acides, l'application de biochar peut provoquer un effet inverse à celui observé dans la présente étude. D'autres travaux révèlent en effet que la concentration en $\mathrm{P}$ en solution dans des sols neutres ou basiques est réduite après un apport de biochar, notamment suite à l'augmentation du $\mathrm{pH}$ et à l'apport massif de cations $\left(\mathrm{Ca}^{2+}\right.$ et $\left.\mathrm{Mg}^{2+}\right)$ qui favorisent la précipitation du $\mathrm{P}$ ou la floculation du P colloïdal (Novak et al., 2009 ; Parvage et al., 2013 ; Xu et al., 2014). 


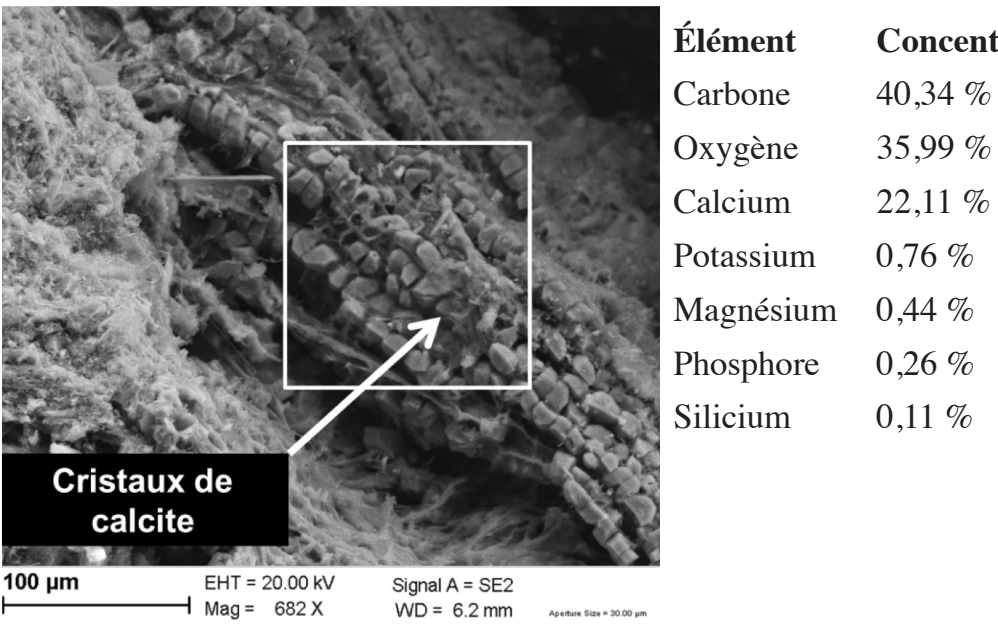

Figure 5. Image prise au microscope électronique à balayage démontrant la présence de cristaux de calcite au sein du biochar de bois - Scanning electron microscope (SEM) image showing the presence of calcite cristals within the wood-derived biochar.

Le tableau indique les concentrations en éléments déterminées semi-quantitativement par EDS au sein de la zone délimitée par le carré blanc - semi-quantitative element concentrations within the area delimited by the white square are shown in the table.
Enfin, l'augmentation de la biodisponibilité $\mathrm{du} P$ dans le traitement BiocharBois-3\% pourrait également résulter d'une plus grande activité biologique induite par l'application de ce biochar, comme le montre la figure 6. Il a par exemple été récemment démontré qu'en favorisant l'activité biologique, notamment celle d'enzymes telles que les phosphatases, l'application de biochar augmentait la biodisponibilité en $\mathrm{P}$ dans les sols en promouvant la décomposition des composés organiques phosphatés (Jin et al., 2016). Cette augmentation de la respiration du sol a été discutée en détails dans un précédent article et résulte principalement de la plus grande présence de composés facilement dégradables au sein du biochar de bois (Houben et al., 2014).

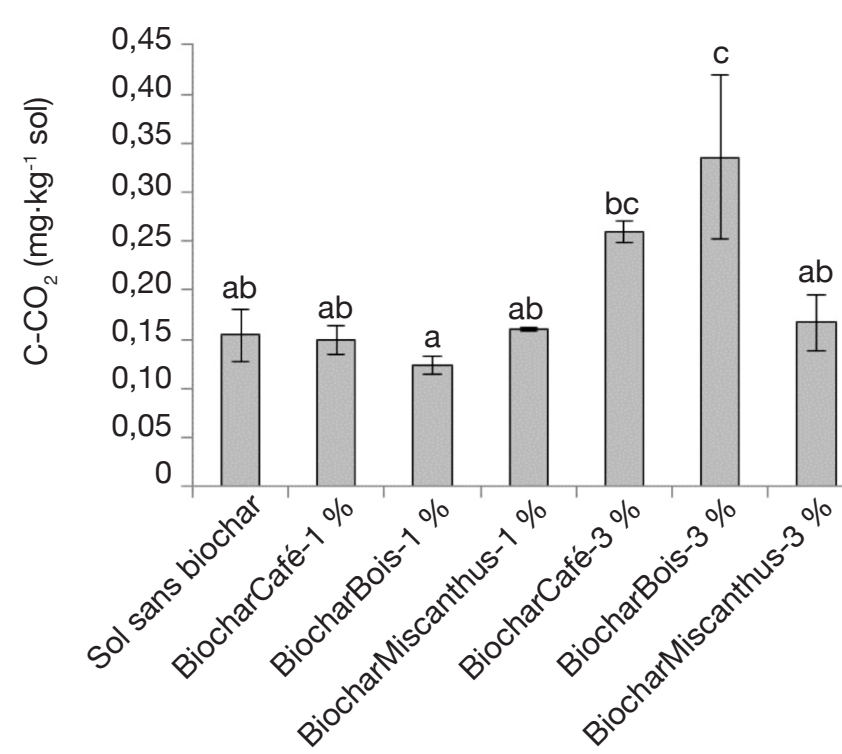

Figure 6. Quantité totale de $\mathrm{C}-\mathrm{CO}_{2}$ émis à l'issue de la période d'incubation pour les différents traitements - Total amount of $\mathrm{CO}_{2}-\mathrm{C}$ released after 76 days of incubation by the untreated soil and in the soil-biochar mixtures.

Les résultats représentent la moyenne de trois réplicats \pm l'erreur standard - results are mean of three replicates \pm standard deviation; les moyennes surmontées de la même lettre alphabétique ne diffèrent pas significativement au seuil de $5 \%$ selon le test de Tukey - columns with same letter do not differ significantly at the $5 \%$ level according to the Tukey's multiple comparison test.

\section{CONCLUSIONS ET IMPLICATIONS}

Le développement d'une agriculture durable implique l'identification et le développement de nouvelles stratégies permettant de limiter le recours aux engrais phosphatés minéraux (Cordell et al., 2009 ; Faucon et al., 2015). La présente étude ambitionnait d'évaluer l'impact de l'application de biochar sur la biodisponibilité du P dans les sols. Résidu solide de la pyrolyse, un procédé de valorisation de la biomasse par conversion thermochimique, le biochar pourrait en effet constituer une solution d'avenir pour la gestion des matières organiques dans les sols car son procédé de production conserve le $\mathrm{P}$ de la matière première. De plus, il est généralement admis que l'application de biochar dans les sols permet de séquestrer du C (Lehmann, 2007) et améliore certaines propriétés agronomiques (Sohi etal.,2010). Nos résultats indiquent cependant que l'application de biochar dans un Luvisol acide n'engendre pas de hausse significative de la biodisponibilité du P, à l'exception du biochar produit à partir de résidus de bois apporté à une dose de $3 \%$. Pour ce dernier traitement, bien que nous n'ayons pas été en mesure d'identifier les mécanismes responsables de l'augmentation de la biodisponibilité du P, il semblerait que celle-ci résulte non seulement d'une libération de $\mathrm{P}$ par le biochar lui-même (effet direct) mais également d'une remobilisation du $\mathrm{P}$ du sol (effet indirect) suite à l'élévation drastique du $\mathrm{pH}(+3,6$ unités par rapport au sol témoin), ainsi qu'à l'augmentation de l'activité biologique. Afin de prédire l'impact du biochar sur la biodisponibilité du P, les futures études devront s'atteler 
à différencier la contribution relative de ces effets, notamment en identifiant la quantité de $\mathrm{P}$ biodisponible apportée par le biochar lui-même. Cette identification nécessitera la mise au point de méthodes permettant de déterminer les différentes formes du $\mathrm{P}$ au sein du biochar et, en particulier, celles alimentant rapidement la solution du sol. Actuellement, la détermination de la biodisponibilité et, plus généralement, de la spéciation $\mathrm{du} \mathrm{P}$ au sein du biochar ne fait toutefois l'objet d'aucun consensus méthodologique (Faucon et al., 2015).

$\mathrm{Eu}$ égard à la variabilité de nos résultats, il nous apparait essentiel de conduire des études complémentaires afin d'accroitre la pertinence et la généricité de l'utilisation du biochar pour une application auprès d'acteurs du développement des filières de recyclage et de fertilisation. Ces études devront notamment viser à distinguer l'effet direct de l'effet indirect de l'application de biochar sur la dynamique $\mathrm{du} \mathrm{P}$, déterminer les matières premières et les doses les plus appropriées pour une fertilisation phosphatée et estimer l'impact de cette stratégie sur la biodisponibilité du $\mathrm{P}$ à plus long terme. L'élucidation de l'effet indirect du biochar sur la remobilisation du $\mathrm{P}$ dans le contexte de la gestion des matières organiques pourrait s'avérer particulièrement intéressante, en particulier dans le contexte wallon étant donné le degré de saturation en $\mathrm{P}$ élevé présenté par la quasi-totalité des sols (Houben et al., 2011 ; Renneson et al., 2015). À l'instar de travaux antérieurs, notre étude souligne également le rôle prépondérant que peut jouer l'effet alcalinisant du biochar sur les processus étudiés. Nous recommandons dès lors que les futures études intègrent systématiquement un témoin chaulé (i.e. un sol dont le $\mathrm{pH}$ a été ajusté à celui mesuré dans le sol après un apport de biochar) afin de déterminer l'importance du rôle joué par cet effet alcalinisant dans l'amélioration des propriétés agronomiques et environnementales potentiellement observée en présence de biochar. Enfin, il convient également de préciser les effets du biochar à plus long terme. Par exemple, les études de Hardy et al. $(2016,2017)$ examinant l'impact d'un enfouissement ancestral de charbon de bois, un matériau très similaire au biochar, sur les propriétés des sols indiquent que l'effet chaulant s'amenuise au fil du temps, suggérant que les mécanismes contrôlant la biodisponibilité du $\mathrm{P}$ à long terme diffèrent de ceux impliqués à court terme.

\section{Bibliographie}

Atienza-Martínez M. et al., 2014. Phosphorus recovery from sewage sludge char ash. Biomass Bioenergy, 65, 42-50.

Azuara M., Kersten S.R.A. \& Kootstra A.M.J., 2013. Recycling phosphorus by fast pyrolysis of pig manure: concentration and extraction of phosphorus combined with formation of value-added pyrolysis products. Biomass Bioenergy, 49, 171-180.
Biederman L.A. \& Harpole W.S., 2013. Biochar and its effects on plant productivity and nutrient cycling: a metaanalysis. GCB Bioenergy, 5, 202-214.

Boulaine J., 2006. Histoire de la fertilisation phosphatée. Étude Gestion Sols, 13, 129-137.

Chan K.Y. \& Xu Z., 2009. Biochar: nutrient properties and their enhancement. In: Lehmann J. \& Joseph S., eds. Biochar for environmental management - Science and technology. London: Earthscan, 67-84.

Chao T.T. \& Sanzolone R.F., 1992. Decomposition techniques. J. Geochem. Explor., 44, 65-106.

Chintala R. et al., 2014. Phosphorus sorption and availability from biochars and soil/biochar mixtures. Clean, 42, 626634.

Cong P.T. \& Merckx R., 2005. Improving phosphorus availability in two upland soils of Vietnam using Tithonia diversifolia H. Plant Soil, 269, 11-23.

Cordell D., Drangert J.-O. \& White S., 2009. The story of phosphorus: global food security and food for thought. Global Environ. Change, 19, 292-305.

DeLuca T.H., MacKenzie M.D. \& Gundale M.J., 2009. Biochar effects on soil nutrient transformations. In: Lehmann J. \& Joseph S., eds. Biochar for environmental management - Science and technology. London: Earthscan, 251-270.

Devau N. et al., 2009. Soil pH controls the environmental availability of phosphorus: experimental and mechanistic modelling approaches. Appl. Geochem., 24, 2163-2174.

Devau N., Le Cadre E., Hinsinger P. \& Gérard F., 2010. A mechanistic model for understanding root-induced chemical changes controlling phosphorus availability. Ann. Bot., 105, 1183-1197.

Faucon M.-P. et al., 2015. Advances and perspectives to improve the phosphorus availability in cropping systems for agroecological phosphorus management. Adv. Agron., 134, 51-79.

Fixen P.E. \& Johnston A.M., 2012. World fertilizer nutrient reserves: a view to the future. J. Sci. Food Agric., 92, 1001-1005.

Gaunt J.L. \& Lehmann J., 2008. Energy balance and emissions associated with biochar sequestration and pyrolysis bioenergy production. Environ. Sci. Technol., 42, 4152-4158.

Gérard F., 2016. Clay minerals, iron/aluminum oxides, and their contribution to phosphate sorption in soils - A myth revisited. Geoderma, 262, 213-226.

Hardy B. et al., 2016. The effect of pre-industrial charcoal kilns on chemical properties of forest soil of Wallonia, Belgium. Eur. J. Soil Sci., 67, 206-216.

Hardy B. et al., 2017. Evaluation of the long-term effect of biochar on properties of temperate agricultural soil at preindustrial charcoal kiln sites in Wallonia, Belgium. Eur. J. Soil Sci., 68, 80-89.

Hass A. et al., 2012. Chicken manure biochar as liming and nutrient source for acid Appalachian soil. J. Environ. Qual., 41, 1096-1106. 
Houba V.J.G., Temminghoff E.J.M., Gaikhorst G.A. \& van Vark W., 2000. Soil analysis procedures using 0.01 M calcium chloride as extraction reagent. Commun. Soil Sci. Plant Anal., 31, 1299-1396.

Houben D., Meunier C., Pereira B. \& Sonnet P., 2011. Predicting the degree of phosphorus saturation using the ammonium acetate-EDTA soil test. Soil Use Manage., 27, 283-293.

Houben D., Evrard L. \& Sonnet P., 2013a. Beneficial effects of biochar application to contaminated soils on the bioavailability of $\mathrm{Cd}, \mathrm{Pb}$ and $\mathrm{Zn}$ and the biomass production of rapeseed (Brassica napus L.). Biomass Bioenergy, 57, 196-204.

Houben D., Evrard L. \& Sonnet P., 2013b. Mobility, bioavailability and $\mathrm{pH}$-dependent leaching of cadmium, zinc and lead in a contaminated soil amended with biochar. Chemosphere, 92, 1450-1457.

Houben D., Sonnet P. \& Cornelis J.-T., 2014. Biochar from Miscanthus: a potential silicon fertilizer. Plant Soil, 374, 871-882.

Houben D. \& Sonnet P., 2015. Impact of biochar and rootinduced changes on metal dynamics in the rhizosphere of Agrostis capillaris and Lupinus albus. Chemosphere, 139, 644-651.

Jeffery S., Verheijen F.G.A., van der Velde M. \& Bastos A.C., 2011. A quantitative review of the effects of biochar application to soils on crop productivity using metaanalysis. Agric. Ecosyst. Environ., 144, 175-187.

Jin Y.etal.,2016. Manure biochar influence upon soil properties, phosphorus distribution and phosphatase activities: a microcosm incubation study. Chemosphere, 142, 128-135.

Joseph S.D. et al., 2010. An investigation into the reactions of biochar in soil. Soil Res., 48, 501-515.

Kookana R.S. et al., 2011. Biochar application to soil: agronomic and environmental benefits and unintended consequences. In: Sparks D.L., ed. Advances in Agronomy. Vol. 112. London: Academic Press, 103-143.

Lehmann J., 2007. A handful of carbon. Nature, 447, 143-144.

Lehmann J., Gaunt J. \& Rondon M., 2006. Biochar sequestration in terrestrial ecosystems - A review. Mitigation Adaptation Strategies Global Change, 11, 395-419.

Lehmann J. \& Joseph S., 2009. Biochar for environmental management - an introduction. In: Lehmann J. \& Joseph S., eds. Biochar for environmental management Science and technology. London: Earthscan, 1-12.

Lehmann J. et al., 2011. Biochar effects on soil biota - A review. Soil Biol. Biochem., 43, 1812-1836.

Lide D.R., 2004. CRC Handbook of chemistry and physics. $85^{\text {th }}$ ed. Boca Raton, FL, USA: CRC Press.

Manyà J.J., 2012. Pyrolysis for biochar purposes: a review to establish current knowledge gaps and research needs. Environ. Sci. Technol., 46, 7939-7954.

McDowell R.W. et al., 2002. Analysis of potentially mobile phosphorus in arable soils using solid state nuclear magnetic resonance. J. Environ. Qual., 31, 450-456.
McDowell R.W. \& Sharpley A.N., 2003. Phosphorus solubility and release kinetics as a function of soil test $\mathrm{P}$ concentration. Geoderma, 112, 143-154.

McDowell R.W., Mahieu N., Brookes P.C. \& Poulton P.R., 2003. Mechanisms of phosphorus solubilisation in a limed soil as a function of pH. Chemosphere, 51, 685-692.

Murrmann R.P. \& Peech M., 1969. Effect of pH on labile and soluble phosphate in soils. Soil Sci. Soc. Am. J., 33, 205.

Novak J.M. et al., 2009. Impact of biochar amendment on fertility of a southeastern coastal plain soil. Soil Sci., 174, 105-112.

Ohno T. \& Crannell B.S., 1996. Green and animal manurederived dissolved organic matter effects on phosphorus sorption. J. Environ. Qual., 25, 1137.

Parvage M.M. et al., 2013. Phosphorus availability in soils amended with wheat residue char. Biol. Fertil. Soils, 49, 245-250.

Rees F., Simonnot M.O. \& Morel J.L., 2014. Short-term effects of biochar on soil heavy metal mobility are controlled by intra-particle diffusion and soil $\mathrm{pH}$ increase. Eur. J. Soil Sci., 65, 149-161.

Renneson M. et al., 2015. Degree of phosphorus saturation in agricultural loamy soils with a near-neutral pH. Eur. J. Soil Sci., 66, 33-41.

Rodella A.A. \& Saboya L.V., 1999. Calibration for conductimetric determination of carbon dioxide. Soil Biol. Biochem., 31, 2059-2060.

Schneider F. \& Haderlein S.B., 2016. Potential effects of biochar on the availability of phosphorus - mechanistic insights. Geoderma, 277, 83-90.

Sohi S.P., Krull E., Lopez-Capel E. \& Bol R., 2010. A review of biochar and its use and function in soil. In: Sparks D.L., ed. Advances in Agronomy. Vol. 105. London: Academic Press, 47-82.

Van Vuuren D.P., Bouwman A.F. \& Beusen A.H.W., 2010. Phosphorus demand for the 1970-2100 period: a scenario analysis of resource depletion. Global Environ. Change, 20, 428-439.

Verheijen F.G.A. et al., 2010. Biochar application to soils a critical scientific review of effects on soil properties, processes and functions. Luxembourg: European Commission.

Xu G., Sun J., Shao H. \& Chang S.X., 2014. Biochar had effects on phosphorus sorption and desorption in three soils with differing acidity. Ecol. Eng., 62, 54-60.

Yaman S., 2004. Pyrolysis of biomass to produce fuels and chemical feedstocks. Energy Convers. Manage., 45, 651-671.

Yuan J.-H., Xu R.-K. \& Zhang H., 2011. The forms of alkalis in the biochar produced from crop residues at different temperatures. Bioresour. Technol., 102, 3488-3497.

Zhai L. et al., 2015. Short-term effects of maize residue biochar on phosphorus availability in two soils with different phosphorus sorption capacities. Biol. Fertil. Soils, 51, 113-122.

(53 réf.) 
